\title{
INFINITE LOOP MAPS AND THE COMPLEX $J$-HOMOMORPHISM
}

\author{
BY VICTOR SNAITH
}

Communicated by P. T. Church, December 23, 1975

\begin{abstract}
We study the complex $J$-homomorphism $j: U \rightarrow S G$ as the composition of two infinite loop maps.
\end{abstract}

1. Introduction. Let $p$ be an odd prime and let $q$ be a prime generating the units of $Z / p^{2}$. All spaces will be $p$-localized. The solution of the Adams conjecture establishes a commutative diagram of fibre sequences.

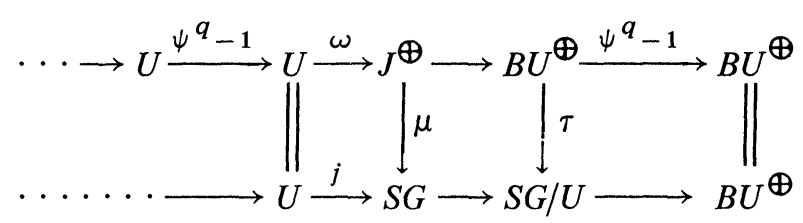

Several, possibly different, $\tau$ have been constructed ([2], [5] and [8]). Given $\tau$, then $\mu$ is unique. The fibre sequences are sequences of infinite loop maps and it is natural to ask whether (1.1) can be extended arbitrarily to the rightthe infinite loop Adams conjecture. By [4] this would be true if $\tau$ were an infinite loop map. These results suggest strongly the validity of the conjecture.

In [2] an $H$-map, $\tau$, is given. If $\mathbf{F}_{q}$ is the field with $q$ elements the finite dimensional vector spaces over $\mathbf{F}_{q}$ under direct sum form a permutative category from which the infinite loopspace $J^{\oplus}$ is constructed by the technique of [1] . Similarly $S G$ is obtained from a category of finite sets under cartesian product. The forgetful functor gives the "discrete models" infinite loop maps $\delta: J \oplus$ $\longrightarrow S G$.

THEOREM 1. If $\tau$ is the map constructed in [2] then $\mu=\delta$ in (1.1).

$J^{\otimes}$ is the infinite loopspace obtained from a category of vector spaces of $\mathbf{F}_{q}$ under tensor product. Assigning to a set the vector space generated by its elements gives $\nu: S G \rightarrow J^{\otimes}$. Define Coker $J^{\otimes}$ by the infinite loop fibering Coker $J^{\otimes} \stackrel{\pi}{\longrightarrow} S G \stackrel{\nu}{\longrightarrow} J^{\otimes}$.

THEOREM 2. $\quad v \circ f: J^{\oplus} \longrightarrow J^{\otimes}$ is a homotopy equivalence for any map $f: J^{\oplus} \rightarrow S G$ such that $f_{\#}$ is nontrivial on $\pi_{2 p-3}$.

AMS (MOS) subject classifications (1970). Primary 55E50, 55F25; Secondary 55D35, $55 \mathrm{~B} 20$.

Key words and phrases. J-homomorphism, infinite loop Adams conjecture, transfer, permutative category, discrete models map. 
TheUREM 3. In (1.1), $j=\delta \circ \omega$.

Combining this with Theorem 2 we easily obtain

THEOREM 4 [9]. There is an equivalence of infinite loopspaces $\delta+\pi$ : $J^{\oplus} \times \operatorname{Coker} J^{\otimes} \rightarrow S G$.

2. If the infinite loop Adams conjecture were true then there would exist an infinite loop map $J^{\oplus} \longrightarrow S G$ satisfying Theorems 1,3 and 4 .

Theorems 2, 3 and 4 can be proved without mentioning $\tau$ at all, i.e. without the solution of the Adams conjecture. For example cf. [7, I] .

Proof of Theorem 3. In [6] a cohomology theory, $A d_{q}^{*}$ is constructed satisfying

$$
\left[X, Z \times J^{\oplus}\right]=A d_{q}^{0}(X)
$$

and giving an infinite loopspace structure to $Z \times J^{\oplus}$ extending the usual one on $J^{\oplus} . A d_{q}^{0}(X)$ has a description in terms of isomorphisms of $Z / q$-vector bundles

$$
\theta: E^{\otimes q} \longrightarrow E \oplus\left(E^{\prime} \otimes N\right)
$$

where $E, E^{\prime}$ are complex vector bundles over $X$ and $N$ is the complex regular representation of $Z / q$. A similar theory constructed from isomorphisms, $\theta$, such that

$$
\mu(\theta): E \stackrel{\text { diag }}{\longrightarrow} E \otimes q \longrightarrow E \oplus\left(E^{\prime} \otimes N\right) \stackrel{\text { proj }}{\longrightarrow} E
$$

is a proper map is also $A d_{q}^{*}$. Sending $\theta$ to the stabilization of $\mu(\theta)$ gives an exponential $H$-map,

$$
\mu: \bigcup_{n \geqslant 0}(n) \times J^{\oplus} \rightarrow \bigcup_{n \geqslant 0} Q_{q} S^{0},
$$

where $Q_{q} S^{0}$ is the set of maps of degree $q^{n}$ in $\Omega^{\infty} S^{\infty}$. It is easy to show explicitly that

$$
\mu \circ \omega=j: U \longrightarrow(0) \times J^{\oplus} \longrightarrow Q_{1} S^{0} \longrightarrow S G .
$$

Also $A d_{q}^{0}$ has a transfer for cyclic coverings which admits an explicit bundle-theoretic description from which it is simple to see that $\mu$ commutes with cyclic covering transfers [3] of the two infinite loopspaces $J^{\oplus}$ and $S G$. Since $\delta$ extends to an exponential $H$-map

$$
\delta: \bigcup_{n \geqslant 0}(n) \times J^{\oplus} \rightarrow \bigcup_{n \geqslant 0} Q_{q} S^{0}
$$

which commutes with transfers for finite coverings, Theorem 3 is a consequence of the following result.

THEOREM 5. There is a unique exponential H-map $\mu: \bigcup_{n \geqslant 0}(n) \times J^{\oplus}$ $\longrightarrow \bigcup_{n \geqslant 0} Q_{q} S^{0}$ which commutes with p-fold cyclic covering transfers and maps (n) $\times J^{\oplus}$ to $Q_{q}{ } S^{0}$. 
Since $\tau$ induces a unique $\mu$ we may also deduce Theorem 3 from Theorem 1 , once we acknowledge the existence of $\tau$.

Proof of TheOrem 1 (cf. [7, II]). $\tau$ is described explicitly in terms of the geometry of fibre bundles of the form $U(n) / N \rightarrow B N \rightarrow B U(n)$. The transfer on $S G / U$ may be extended to the space $U_{n \geqslant 0}(n) \times(S G / U)$. Furthermore $\tau$ may be extended to an $H$-map

$$
\bar{\tau}: \bigcup_{n \geqslant 0}(n) \times B U^{\oplus} \rightarrow \bigcup_{n \geqslant 0}(n) \times(S G / U),
$$

which maps $(n) \times B U^{\oplus}$ to $(n) \times(S G / U)$. Also $\bar{\tau}$ commutes with $p$-fold cyclic covering transfers. The proof of Theorem 1 is completed by means of the analogue of Theorem 5 for $H$-maps $\bigcup_{n \geqslant 0}(n) \times J^{\oplus} \longrightarrow \bigcup_{n \geqslant 0}(n) \times(S G / U)$.

\section{REFERENCES}

1. D. W. Anderson, Simplicial $K$-theory and generalized cohomology theories (to appear).

2. J. C. Becker and D. H. Gottlieb, The transfer map and fibre bundles, Topology 14 (1975), 1-12.

3. D. S. Kahn and S. B. Priddy, Applications of the transfer to stable homotopy theory, Bull. Amer. Math. Soc. 78 (1972), 981-987. MR 46 \#8220.

4. I. Madsen, V. Snaith and J. Tornehave, Homomorphisms of spectra and bundle theories, Aarhus Univ. Preprint Ser., no. 31, 1974/75.

5. D. G. Quillen, The Adams conjecture, Topology 10 (1971), 67-80. MR 43 \#5525.

6. R. M. Seymour, Vector bundles invariant under the Adams operations, Quart. J. Math. Oxford Ser. (2) (1974), 395-414.

7. V. P. Snaith, The complex J-homomorphism. I, II, Proc. London Math. Soc. (to appear).

8. D. Sullivan, Genetics of homotopy theory and the Adams conjecture, Ann. of Math. (2) 100 (1974), 1-80.

9. J. Tornehave, The splitting of spherical fibration theory at odd primes, Aarhus Univ. Preprint Ser., no. 28, $1972 / 1973$.

DIVISION OF MATHEMATICAL SCIENCES, PURDUE UNIVERSITY, WEST LAFAYETTE, INDIANA 47907 